CASE REPORT

\title{
Late onset group B streptococcal disease manifested by isolated cervical lymphadenitis
}

\author{
K Fluegge, P Greiner, R Berner
}

Arch Dis Child 2003;88:1019-1020

Sepsis and meningitis are the major clinical manifestations of group B streptococcal (GBS) infections in neonates, but GBS can cause a wide spectrum of presentations ranging from asymtomatic bacteraemia to fulminate septicaemia and shock. To our knowledge this is the first report of isolated neonatal lymphadenitis as a manifestation of late onset GBS disease.

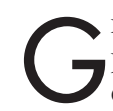
roup B streptococcus (GBS) is one of the leading pathogens of neonatal infection of both early and late onset. $^{1-3}$ Early onset sepsis is typically related to vaginal carriage of the mother and vertical transmission during birth. The origin of late onset disease is less clear. It is believed to be transmitted both vertically and horizontally from maternal and nosocomial sources, or to be the result of persistent colonisation. ${ }^{3}$ Whereas sepsis and meningitis are the most common clinical manifestations, GBS can cause a wide spectrum of focal infections such as osteomyelitis, ethmoiditis, empyema, conjunctivitis, and cellulitis-adenitis, or non-focal disease ranging from asymptomatic bacteraemia to fulminant septic shock. To our knowledge this is the first report of isolated lymphadenitis as a manifestation of late onset GBS disease in a young infant.

\section{CASE REPORT}

A $3760 \mathrm{~g}$ male infant was born to a healthy 26 year old mother at 41 weeks gestational age. Due to alterations of the cardiotocograph, cesarean section had to be performed; the immediate neonatal period, however, was uneventful. The infant was discharged from the hospital at the age of 8 days. The boy was breast fed and developed well until the age of 28 days when he became irritable. The consulting paediatrician noticed a submandibular mass on the right side as well as increasing irritability. The infant was immediately referred to the hospital for further diagnosis and treatment. On admission, physical examination showed an irritable infant with a rectal temperature of $38.4{ }^{\circ} \mathrm{C}$. He presented with a slightly warm mass in the right submandibular region, revealing a localised lymphadenopathy which had increased in size within a few hours. There were no signs of ipsilateral local infections such as cellulitis, parotitis, or otitis media. Ultrasonography of the region revealed multiple enlarged lymph nodes ranging from 0.7 to $1.1 \mathrm{~cm}$ diameter in size, without any abscess formation or signs of cellulitis. Otherwise, there were no remarkable physical findings. Initial laboratory data revealed a white blood cell count (WBC) of $4.6 \times 10^{9} / 1$ with a significant left shift (34\% band forms and $65 \%$ neutrophils); C reactive protein (CRP) serum concentration was $1.0 \mathrm{mg} / \mathrm{dl}$. A blood culture was obtained and empirical antibiotic therapy with cefotiam, a parenteral cephalosporin of the cefuroxime group, was started. Within 24 hours the infant became afebrile and less irritable. After a maximum of $3.1 \mathrm{mg} / \mathrm{dl}$ on day 3, CRP normalised within 12 days after admission. The further clinical course was uneventful. Antibiotic therapy was continued for a total of 14 days, while the submandibular lymphadenitis had resolved a few days after initiation of antibiotic treatment.

Twenty four hours after admission the blood culture yielded growth of GBS; antibiotic therapy was switched to penicillin and netilmicin on day 3, and was continued for 12 and 5 days, respectively. A lumbar puncture was performed on day 2 after antibiotic treatment was started to exclude meningeal involvement; there was no inflammatory cellular cerebrospinal fluid (CSF) reaction (1 leucocyte/ $\mu$ l), and CSF cultures remained sterile.

Although the mother of our patient showed no signs of mastitis, a sample of the breast milk was analysed, but results of the bacterial cultures were negative. Thus, the source of infection remained unidentified.

\section{DISCUSSION}

Cellulitis-adenitis is a well known but rare presentation of GBS infection in neonates and infants. ${ }^{45}$ To our knowledge, it has been described in 32 infants. ${ }^{5-15}$ Although the presenting spectrum is wide, there were some common features in all reported cases such as poor feeding, irritability, fever, and localised swelling of the skin. The submandibular region has been the most common site of cellulitis and lymph node enlargement. In 1982, Baker reported GBS cellulitis in seven infants, localised mainly in the submandibular region. Since all patients had significant focal adenitis, the term cellulitisadenitis was established. The exact pathogenesis is not yet clear. One hypothesis postulates that lymphatic spread from a primary focus such as otitis media may account for cellulitis and subsequent adenitis. ${ }^{5}$ Since there were no signs of cellulitis in our patient, it is very likely that lymphatic spread of GBS was not the causative event. Another hypothesis postulates the infant as a carrier of GBS, followed by bloodstream invasion of a pathogenic bacterial strain and subsequent seeding in the neck area. ${ }^{15}$ Since previously reported cases found cellulitis always associated with cervical lymphadenitis, our case might provide some additional evidence for bacteraemia as the primary pathogenetic event.

Because cellulitis-adenitis is an uncommon manifestation of GBS disease, the data concerning CNS involvement are scarce. Coexistent meningitis occurs but has been described as rare. ${ }^{16}$ This observation may be due to the small number of cases studied. ${ }^{6}$ In contrast, other focal infections such as osteomyelitis have frequently been associated with meningitis. ${ }^{17}{ }^{18}$ This association is not surprising since focal infections in very young infants are usually preceded by bacteraemia, and therefore the potential for seeding of the CNS clearly exists. The origin of bloodstream infection in late onset disease is not clearly elucidated. GBS disease associated with ingestion of infected breast milk has been described. ${ }^{19} 20$ Breast milk was analysed in our patient but GBS cultures 
remained sterile. Therefore, the route of transmission via infected breast milk does not seem probable.

This case of isolated GBS lymphadenitis shares similarities with the cellulitis-adenitis syndrome, which has been noted as a late onset manifestation of GBS infection in preterm and term, predominantly male infants, aged 0 to 10 weeks (median 4.5 weeks), who have non-specific signs of systemic infection including fever and irritability. ${ }^{5-7} 101521$ The case reported here is unusual because of the isolated localised lymphadenitis and the lack of obvious cellulitis. From the clinical point of view, GBS should be considered as a possible aetiologic agent in young infants presenting with nonspecific signs of systemic infection and localised lymphadenitis. Isolated lymphadenitis as well as cellulitis may be a readily evident indicator of underlying bacteraemia, and therefore can serve as a valuable clinical clue. Physicians need to be aware of unusual forms of neonatal GBS infection and possible meningeal involvement as a manifestation of late onset GBS invasive infection, resulting in a different diagnostic course and treatment.

\section{Authors' affiliations}

K Fluegge, P Greiner, R Berner, Department of Pediatrics, University of Freiburg, Freiburg, Germany

Correspondence to: Dr R Berner, University Children's Hospital, Mathildenstrasse 1, D-79106 Freiburg, Germany; berner@ kikli.ukl.uni-freiburg.de

Accepted 10 March 2003

\section{REFERENCES}

1 Berner R. Group B streptococci during pregnancy and infancy. Curr Opin Infect Dis 2002;15:307-13.
2 Gladstone IM, Ehrenkranz RA, Edberg SC, et al. A ten-year review of neonatal sepsis and comparison with the previous fifty-year experience. Pediatr Infect Dis J 1990;9:819-25.

3 Fernandez M, Rench MA, Albanyan EA, et al. Failure of rifampin to eradicate group $B$ streptococcal colonization in infants. Pediatr Infect Dis J $2001 ; 20: 371-6$.

4 Howard JB, McCracken GH. The spectrum of group B streptococcal infections in infancy. Am J Dis Child 1974;128:815-18.

5 Baker CJ. Group B streptococcal cellulitis-adenitis in infants. Am J Dis Child 1982;136:631-3.

6 Hauger SB. Facial cellulitis: an early indicator of group B streptococcal bacteremia. Pediatrics 1981;67:376-7.

7 Pathak A, Hwu HH. Group B streptococcal cellulitis. South Med J 1985:78:67-8

8 Brady MT. Cellulitis of the penis and scrotum due to group B streptococcus. J Urol 1987; 137:736-7

9 Cleary TG, Kohl S. Overwhelming infection with group B beta-hemolytic streptococcus associated with circumcision. Pediatrics 1979;64:301-3.

10 Asmar BI. Neonatal retropharyngeal cellulitis due to group B streptococcus. Clin Pediatr (Phila) 1987;26:183-5.

11 Rand TH. Group B streptococcal cellulitis in infants: a disease modified by prior antibiotic therapy or hospitalization? Pediatrics 1988;81:63-5.

12 Rathore MH. Group B streptococcal cellulitis and adenitis concurrent with meningitis. Clin Pediatr (Phila) 1989;28:411.

13 Barton LL, Ramsey RA, Raval DS. Neonatal group B streptococcal cellulitisadenitis. Pediatr Dermatol 1993; 10:58-60.

14 Doedens RA, Miedema CJ, Oetomo SB, et al. Atypical cellulitis due to group B streptococcus. Scand J Infect Dis 1995;27:399-400.

15 Patamasucon P, Siegel JD, McCracken GH Jr. Streptococcal submandibular cellulitis in young infants. Pediatrics 1981;67:378-80.

16 Marcy SM, Overturf GD. Infections of the skin and subcutaneous tissue. In: Remington JS, Klein JO, eds. Infectious diseases of the fetus and newborn infant. Philadelphia, PA: WB Saunders, 1995:958-64.

17 Klein JO, Marcy SM. Bacterial sepsis and meningitis. In: Remington JS, Klein JO, eds. Infectious diseases of the fetus and newborn infant. Philadelphia, PA: WB Saunders, 1995:835-90.

18 Broughton RA, Edwards MS, Haffar A, et al. Unusual manifestations of neonatal group B streptococcal osteomyelitis. Pediatr Infect Dis J 1982;1:410-12.

19 Kenny JF. Recurrent group B streptococcal disease in an infant associated with the ingestion of infected mother's milk. J Pediatr 1977;91:158-9.

20 Olver WJ, Bond DW, Boswell TC, et al. Neonatal group B streptococcal disease associated with infected breast milk. Arch Dis Child Fetal Neonatal Ed 2000;83:F48-9.

21 Ruiz-Gomez D, Tarpay MM, Riley HD. Recurrent group B streptococcal infections: report of three cases. Scand J Infect Dis 1979;11:35-8. 